\title{
Investigation of Cue-based Aggregation in Static and Dynamic Environments with a Mobile Robot Swarm
}

\author{
Farshad Arvin ${ }^{1,2} \quad$ Ali Emre Turgut ${ }^{3} \quad$ Tomáš Krajník ${ }^{4} \quad$ Shigang Yue \\ ${ }^{1}$ School of Electrical and Electronic Engineering, \\ The University of Manchester, M13 9PL, \\ Manchester, United Kingdom \\ 2 Computational Intelligence Laboratory (CIL), School of Computer Science, \\ University of Lincoln, LN6 7TS, United Kingdom \\ 3 Mechanical Engineering Department \\ Middle East Technical University (METU) \\ 06800 Ankara, Turkey \\ ${ }^{4}$ Lincoln Centre for Autonomous Systems (L-CAS), \\ University of Lincoln, LN6 7TS, United Kingdom
}

Contact person: Shigang Yue

School of Computer Science

University of Lincoln, Brayford Pool,

Lincoln, LN6 7TS, United Kingdom

E-mail: syue@lincoln.ac.uk 


\begin{abstract}
Aggregation is one of the most fundamental behaviors that has been studied in swarm robotic researches for more than two decades. The studies in biology revealed that environment is a preeminent factor in especially cue-based aggregation that can be defined as aggregation at a particular location which is a heat or a light source acting as a cue indicating an optimal zone. In swarm robotics, studies on cue-based aggregation mainly focused on different methods of aggregation and different parameters such as population size. Although of utmost importance, environmental effects on aggregation performance have not been studied systematically. In this paper, we study the effects of different environmental factors; size, texture and number of cues in a static setting and moving cues in a dynamic setting using real robots. We used aggregation time and size of the aggregate as the two metrics to measure aggregation performance. We performed real robot experiments with different population sizes and evaluated the performance of aggregation using the defined metrics. We also proposed a probabilistic aggregation model and predicted the aggregation performance accurately in most of the settings. The results of the experiments show that environmental conditions affect the aggregation performance considerably and have to be studied in depth.
\end{abstract}

Keywords: Swarm Robotics, Aggregation, Collective Behavior, Cue-based aggregation 


\section{Introduction}

Environment plays a crucial role in the daily routine and life cycle of all animals. Animals, their nests, behaviors and nutrition habits cannot be thought independent of the environment they live in. When we consider social animals such as ants, bees and termites environment becomes even more important in their daily routine. All the decisions they make are based on social interactions with their nest-mates and the state of the environment (Amé, Halloy, Rivault, Detrain, \& Deneubourg, 2006). Environment also serves as a medium for intraspecific communication (stigmergy) that is known to be a very effective way of communication in unstructured and complex environments (Schmickl \& Crailsheim, 2004).

Aggregation is a widely observed phenomenon in social animals especially in social insects (Grünbaum \& Okubo, 1994). It can be defined as gathering of individuals into a single aggregate at a particular location. Aggregation behavior can be observed from amoeba (Rappel, Nicol, Sarkissian, Levine, \& Loomis, 1999) to insects and to other animals (Camazine et al., 2001). Animals in an aggregate gain additional capabilities such as forming a spore-bearing structure by slime mold (Bonner, 1944), building a nest by termites (Parrish \& Edelstein-Keshet, 1999) or protection against predators (Johannesen, Dunn, \& Morrell, 2014; Morrell \& James, 2008).

Two different types of aggregation mechanisms are observed in nature: cue-based and selforganized (Camazine et al., 2001). In cue-based aggregation, animals aggregate on an external cue that is known to be an optimal zone for their survival; such as high temperature or high humidity zone for flies (Frank, Jouandet, Kearney, Macpherson, \& Gallio, 2015). Self-organized aggregation does not require any external cues. Animals aggregate on some locations without any particular preference to their environmental conditions (Garnier, Gautrais, Asadpour, Jost, \& Theraulaz, 2009).

Cue-based and self-organized aggregation have been studied in swarm robotics for more than two decades (Şahin, Girgin, Bayındır, \& Turgut, 2008; Brambilla, Ferrante, Birattari, \& Dorigo, 2013; Bayındır, 2016). In cue-based aggregation, which is the main topic of this paper, one of the seminal works is due to Schmickl et al. (Schmickl, Thenius, et al., 2009). Inspired by honeybee aggregation in which bees aggregate on optimal temperature zones (Heran, 1952), Kernbach et al. (Kernbach, Thenius, Kernbach, \& Schmickl, 2009) proposed a method known as BEECLUST for robot swarms. In BEECLUST, robots perform a random walk, and after a collision with another robot, they wait for a particular amount of time directly proportional to the intensity of the light 
in the environment and then they continue doing a random walk. Many robots encountering with many others cause the swarm to aggregate on the optimal zone defined by the intensity of the light. Follow up works on BEECLUST mainly focused on: (1) modifications of parameters of BEECLUST to improve its performance (Arvin, Samsudin, Ramli, \& Bekravi, 2011; Arvin, Turgut, Bellotto, \& Yue, 2014), (2) derivation of simpler aggregation models based on systematic honeybee experiments (Schmickl \& Hamann, 2011), (3) fuzzy-based aggregation methods for better aggregation performance (Arvin, Turgut, Bazyari, et al., 2014; Arvin, Turgut, \& Yue, 2012), and (4) heterogeneity in behaviors (Kengyel et al., 2015).

Although of utmost importance, to the best of our knowledge little has been done to study the effects of environment on aggregation. In this paper, we present a detailed study on effects of environmental changes on performance of a swarm system. We investigate different types of environments - static and dynamic - to check the influence of the changes on the performance of the bio-inspired aggregation mechanism based on the state-of-the-art BEECLUST algorithm.

\section{Related Work}

Study on honeybees' thermotactic aggregation behavior is an early work on aggregation in biology (Heran, 1952) which showed that young honeybees tend to aggregate at an optimal zone with temperature between $34^{\circ}$ and $38^{\circ} \mathrm{C}$ in a hive. The study revealed that bees follow a simple mechanism to form an aggregate based on two phases: performing a random walk until another bee is encountered and when encountered waiting for a certain amount of time based on the ambient temperature. Szopek et al. (Szopek, Schmickl, Thenius, Radspieler, \& Crailsheim, 2013) studied the collective decision making of honeybees, which leads to thermotaxis-based aggregation at the optimal zone in a hive with a more systematic way. Their study revealed that a large group can find an optimal zone faster than the small size swarm. The results also showed that the group behavior is scalable and robust.

In another study (Raveh, Vogt, Montavon, \& Kölliker, 2014), Raveh et al. showed that earwigs (Forficula auricularia) prefer to form aggregates with their relatives rather than other earwigs. This behavior helps to reduce the risk of competition between the individuals. In another interesting study (Broly, Devigne, Deneubourg, \& Devigne, 2014), Broly et al. showed that aggregation helps woodlice colony (Isopoda: Oniscidea) to reduce water loss hence increase the survival rate of the colony. In case of mammals, a recent study on sea lions (Liwanag, Oraze1, Costa1, \& Williams, 
2014) revealed that sea lions tend to gather and form an aggregate when the ambient temperature reaches critical values. Aggregation helps them to decrease the heat transfer rate, hence keep their body temperature at the optimal level with lower energy loss. Therefore, during cold seasons, most of lions join the aggregate tightly instead of resting alone. Jeanson et al. studied cockroach (Blattella germanica) aggregation in a homogeneous environment (Jeanson et al., 2005). They showed that the probability of a cockroach to stop and wait in an aggregate depends on the size of aggregate. The bigger it is, the longer the waiting time is. On the contrary, Amé et al. (Amé et al., 2006) studied cockroach aggregation in a heterogeneous environment. Using two identical plastic shelters in an arena, they showed that cockroaches prefer to aggregate and rest under dark shelters. They figured out that the probabilities to join and to leave an aggregate are low when the population of the shelter is large. Although, this seems contrary to Jeanson et al. (Jeanson et al., 2005), it is not. In fact, larger aggregate reduces the probability of having access to the cue, hence this forms a negative feedback mechanism.

In swarm robotics (Brambilla et al., 2013), self-organized aggregation has been performed in various studies. Trianni et al. (Trianni, Groß, Labella, Şahin, \& Dorigo, 2003) presented an aggregation behavior using artificial evolution in two different settings: static and dynamic. In the static setting, when robots form an aggregate, they are not allowed to leave it, whereas in the dynamic setting, robots are allowed to leave the aggregate and join the other aggregates in the environment. In the static setting, it is observed that increasing the population size can result in formation of many separate aggregates. In the latter setting, robots in smaller aggregates have the chance to leave them and join the other ones, which finally results in formation of a single large aggregate. In another study, Soysal and Şahin (Soysal \& Şahin, 2005) proposed a probabilistic aggregation mechanism based on simple behaviors as: obstacle avoidance, approach to an aggregate, repel from an aggregate, and wait. Performance of the system was investigated using various parameters including control strategies, time, and arena configuration. In a follow-up work (Soysal, Bahçeci, \& Şahin, 2007), they also studied these parameters in aggregation using artificial evolution. In another study, Halloy et al. (Halloy et al., 2007) studied the aggregation behavior of a mixed group of robots and cockroaches in a two-shelter arena. The results revealed that the mixed group aggregated under the darkest shelter as expected. In a similar study, Garnier et al. (Garnier et al., 2008, 2009) used a miniature robot platform and implemented the behavioral model of cockroaches as proposed in (Jeanson et al., 2005). They were able to mimic the 
aggregation behavior of cockroaches with robots in similar experimental settings as in (Jeanson et al., 2005). Campo et al. (Campo, Garnier, Dédriche, Zekkri, \& Dorigo, 2011) proposed a collective decision making mechanism, which is based on the behavior of cockroaches, to discriminate between two different quality sources. The aim of the robots is to find the source that is the smallest, yet that can encapsulate the whole swarm. The experiments showed that the swarm was able to aggregate at the optimal source location and an increase in population size improved the performance of the swarm. In a recent study, Gauci et al. (Gauci, Chen, Li, Dodd, \& Groß, 2014) proposed a self-organized aggregation mechanism with memory-less mobile robots with a binary sensor. The control mechanism includes: i) rotating on a spot when there is another robot and ii) circular backward movement when no other robot is detected. The results of simulated and real robot experiments showed that, robots tend to make a single aggregate using the proposed simple mechanism. However, to accomplish aggregation, the the binary sensor had to be able to detecto other robots at a long range.

Kube and Zhang (Kube \& Zhang, 1993) have performed one of the earliest studies in cue-based aggregation in swarm robotics. They proposed a collective transport scenario in which robots first aggregate around an object with a light source, and then push that object together. The aggregation method, which was used in that study is based on simple behaviors and does not rely on any explicit communication. To control the size of an aggregate in a cue-based aggregation scenario, Holland and Melhuish (Holland \& Melhuish, 1997) proposed a mechanism, in which robots first aggregate around an infra-red transmitter, and then start to emit sound both synchronously and randomly. Therefore, each robot is able to estimate the aggregate size using the sound signal strength and decide to join and leave the aggregate accordingly. Mermoud et al. (Mermoud, Matthey, Evans, \& Martinoli, 2010) used aggregation in a cue-based setting to enable a collective decision mechanism. Using a probabilistic aggregation method similar to the one in (Soysal \& Şahin, 2005), robots first aggregate on a spot that could be either a bad spot (meaning that it should be destroyed) or a good spot (meaning that nothing should be done) and then they decide collectively whether to destroy or keep the spot intact. They showed that aggregation helps the robots to interact and communicate, which in turn helps them to make correct decisions under uncertainty due to noisy sensing. Francesca et al. (Francesca, Brambilla, Trianni, Dorigo, \& Birattari, 2012) implemented the decision making strategy which cockroaches use in finding a resting shelter when there are more than one. They used a similar experimental setup which was 
proposed in (Amé et al., 2006). The results showed that, the probability of leaving an aggregate relies on the population and the capacity of the shelter. In a recent study (Schmickl \& Hamann, 2011), Schmickl and Hamann worked on the aggregation of young bees as in (Heran, 1952) in a more systematic way. The results of real bee and robot experiments showed that bees follow a very simple set of behaviors for aggregation as: (i) A bee performs correlated a random walk. (ii) When a bee hits a wall, it avoids the wall and then continues to perform a random walk. (iii) When a bee encounters another bee, it stops and waits for a certain amount of time. Waiting time is directly proportional to the temperature of the spot. When the waiting time is over, the bee continues to perform a random walk.

In another study (Kernbach et al., 2009), Kernbach et al. proposed an aggregation method called BEECLUST, which is based on honeybee aggregation as in (Schmickl \& Hamann, 2011). The algorithm is based on robot-to-robot collisions as opposed to bee-to-bee encounters. In their setting, they assumed that there is a light source in the environment, which is used to crate a light gradient. Robots are required to aggregate on the zone where the intensity of the light is the highest. Each robot performs a random walk and stops when it encounters another robot. The waiting time of the robot depends on the intensity of the light where it stopped. The more the intensity, the longer it waits. After the waiting time is over, the robot turns to a random direction and restarts to perform a random walk. Through experiments they showed that robots are able to aggregate on the optimal zone. In a follow-up study (Schmickl, Thenius, et al., 2009), Schmickl et al. proposed two types of experiments. One is the static experiments in which there is a single light source as in (Kernbach et al., 2009) and the other is the dynamic experiments in which there are two light sources with different intensities and the intensities of the sources are changed during an experiment. Through systematic experiments, they showed that as in (Kernbach et al., 2009), robots were able to aggregate on the optimal zone in static experiments. Whereas, in dynamic experiments, robots are able to aggregate close to the highest intensity source and when the intensities of the two sources are switched during the experiment, robots are able to leave the previously formed aggregate and form a new aggregate under the recent optimal zone.

Previously, we studied the effects of the different interactions among a group of robots and their decision making strategies. In (Arvin et al., 2011), we proposed two modifications on BEECLUST in order to increase its performance. One is the dynamic velocity in which robots are allowed to select three different speeds based on intensity of light; higher intensity results in slower speed 
and vice versa. The other modification is the comparative waiting time. The waiting time of a robot increases in the presence of the other robots or aggregates. Both simulation-based and real robot experiments were conducted and results showed that both methods improve aggregation performance. In addition, we studied the effects of turning angle and its calculation methods on the performance of the swarm aggregation (Arvin, Turgut, Bellotto, \& Yue, 2014). In that study, we compared the performance of two proposed aggregation algorithms - vector averaging and naïve - with BEECLUST. The results showed that the proposed strategies outperform BEECLUST method due to additional environmental perception. In a recent study (Arvin, Turgut, Bazyari, et al., 2014), we introduced a fuzzy-based decision making mechanism in swarm aggregation and showed that the proposed method significantly improves the performance of aggregation using real-robot and computer-based simulations (Arvin et al., 2012).

The rest of this paper is organized as follows. In Section 3, we introduce the aggregation method. Following that in Section 4, we introduce the proposed probabilistic model. In Section 5 , we explain the realization of aggregation with real robots. In Section 6 , we discuss the different experimental configurations and different experimental settings. In Section 7, we discuss results of the experiments in different settings. Finally, in Sections 8 and 9, we discuss the future research directions and make a conclusion of the study.

\section{Aggregation Method}

We use the state-of-the-art BEECLUST method (Schmickl, Thenius, et al., 2009). Fig. 1 shows the flowchart of the aggregation method. In this method, a robot moves forward continuously in the environment. When it encounters an object, it checks whether the object is an obstacle or another robot. If it is an obstacle, the robot avoids the obstacle and continues to move forward. If not, it stops and waits for a particular amount of time, the waiting time, $w(t)$. The waiting time is a function of the ambient light intensity (Schmickl, Thenius, et al., 2009), which is estimated by the following formula:

$$
w(t)=\frac{60 S(t)^{2}}{S(t)^{2}+5000},
$$

where $S$ is the illuminance captured by the light sensor varying linearly from 0 and 255 corresponding to 0 lux and 600 lux. After the waiting time is over, the robot rotates $\phi$ degrees and continues 


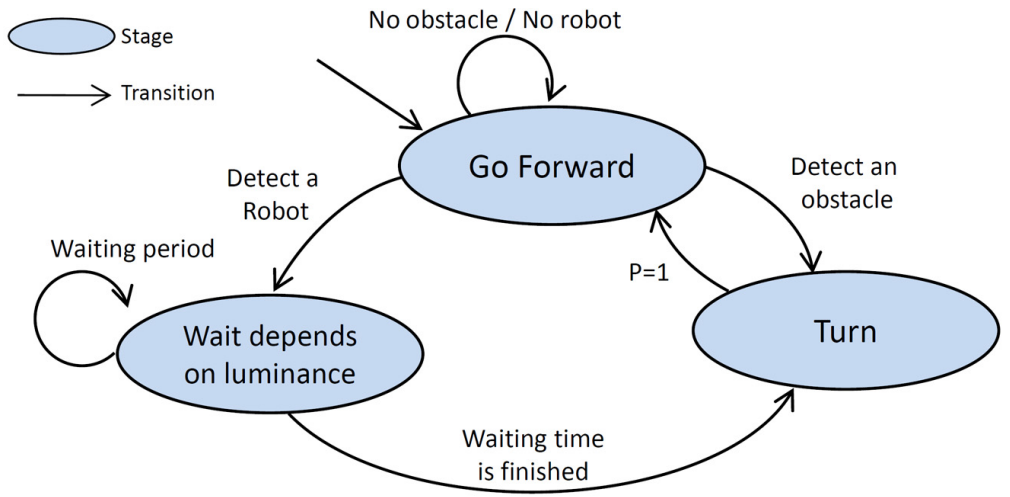

Figure 1: Finite state automaton that shows the robots' behavior in BEECLUST.

to move forward. $\phi$ is a random variable drawn from a uniformly distributed set of angles in the range $\left[-180^{\circ}, 180^{\circ}\right]$.

\section{Probabilistic Modeling of Aggregation}

Stochastic characteristic of aggregation induce to use a probabilistic modeling scheme. To this end, several probabilistic models have been proposed in swarm robotics (Martinoli, Ijspeert, \& Mondada, 1999; Lerman, Galstyan, Martinoli, \& Ijspeert, 2001; Correll \& Martinoli, 2007). Soysal and Şahin (Soysal \& Şahin, 2007) proposed a macroscopic model of an aggregation behavior, which is able to predict the final distribution of the system. Bayındır and Şahin (Bayindir \& Şahin, 2009) proposed a macroscopic model for a self-organized aggregation using probabilistic finite state automata, which could depict the behavior of swarm system appropriately. Hamann (Hamann, 2008) modeled the collective behavior of robots in a cue-based aggregation using a Langevin equation. Schmickl et al. (Schmickl, Hamann, Worn, \& Crailsheim, 2009) proposed a macroscopic modeling of the cue-based aggregation using Stock \& Flow model. In our previous work (Arvin, Attar, Turgut, \& Yue, 2015), we proposed a mathematical model using a power-law equation to predict the aggregate size over time.

In this work, to model the influence of the environmental parameters on the swarm behavior, we use a rate equation that represents the three processes that influence the size of an aggregate in single cue experiments. The equation is based on the probabilities of individual robots joining and leaving the aggregate during a given time interval. The rate of change of the number of aggregated 
robots, $n_{a}$, can be expressed by means of these probabilities as:

$$
\dot{n_{a}}=\frac{\mathrm{d} n_{a}}{\mathrm{~d} t}=n_{f}\left(2 p_{m}+p_{j}\right)-n_{a} p_{l}
$$

where $p_{j}$ is the probability that a robot joins the aggregate, $p_{m}$ represents the probability that two non-aggregated robots meet on the cue, $p_{l}$ represents the chance that an aggregated robot leaves the aggregate and $n_{f}$ is the number of non-aggregated (free) robots. To calculate $p_{j}$ and $p_{m}$, we have to find the chance that one robot detects another one during a given time interval. We based our approximation on an area that a single robot sweeps during a unit of time. Given that radius of the sensory system is $r_{s}$ and the robot radius is $r_{r}$, two robots detect each other if their centers become closer than $r_{s}+r_{r}$. This means that during one second of movement with a speed of $v_{r}$, a robot sweeps an area equal to $a_{s}=\left(r_{s}+r_{r}\right) v_{r}$. Given that the density of the non-aggregated robots excluding the subject robot is homogeneous and equal to $\left(n_{f}-1\right) / a_{a}$, we can calculate the probability $p_{m}$ that two non-aggregated robots meet on the cue as:

$$
p_{m}=a_{s} \frac{n_{f}-1}{a_{a}} \frac{a_{c}}{a_{a}}=\left(n_{f}-1\right) \frac{a_{c} a_{s}}{a_{a}^{2}}
$$

where $a_{c}$ is the area of the cue and $a_{a}$ is the area of the arena.

Similarly, we can calculate a probability $p_{j}$ that a non-aggregated robot meets an aggregated one as:

$$
p_{j}=a_{s} \frac{n_{a}}{a_{c}} \frac{a_{c}}{a_{a}}=n_{a} \frac{a_{s}}{a_{a}}
$$

where $n_{a}$ is the number of aggregated robots, the $n_{a} / a_{c}$ is the density of the robots on the cue and $a_{c} / a_{a}$ equals to the probability that the given robot is on the cue.

To roughly estimate a probability that a robot leaves the aggregate, we take into account the waiting time $w$ and the chance that it will not encounter another aggregated robot on the cue while leaving as:

$$
p_{l}=\frac{1}{2 w}\left(1-\frac{n_{a} a_{s}}{a_{c}} \frac{r_{c}}{2 v_{r}}\right)
$$

where $r_{c} /\left(2 v_{r}\right)$ represents an average time it takes to leave the cue. A robot is also assumed to leave the aggregate when the dynamic cue on which the robots aggregated moves away. This means that the probability $p_{l}$ is increased by the chance that a robot has been in an area that the cue 
left, leading to:

$$
p_{l}=\frac{1}{2 w}\left(1-\frac{n_{a} a_{s}}{a_{c}} \frac{r_{c}}{2 v_{r}}\right)+\frac{v_{c}}{\pi r_{c}}
$$

where $v_{c}$ is the velocity of the dynamic/moving cue (see Section 6.2) and $r_{c}$ is its radius. Combining equations $(3,4,6)$ allows us to express Eq.(2) as:

$$
\dot{n}_{a}=n_{f} \frac{a_{s}}{a_{a}}\left(2\left(n_{f}-1\right) \frac{a_{c}}{a_{a}}+n_{a}\right)-\frac{n_{a}}{2 w}\left(1-\frac{n_{a} a_{s} r_{c}}{2 a_{c} v_{r}}\right)-\frac{n_{a} v_{c}}{\pi r_{c}} .
$$

Taking into account that $n_{f}+n_{a}=n$, the rate of change $i_{a}$ can be fully expressed as a function of $n_{a}$, which allows us to calculate how the number of aggregated robots would change over time. Thus, it can be used to estimate the influence of certain parameters on the swarm behavior. Since a full analytic solution of this equation is beyond the scope of this paper, we created a Simulink model shown in Fig. 13 (see Appendix A) that allows us to change the model's parameters and study their influence qualitatively.

The proposed probabilistic model suggests that the rate at which the swarm aggregates increases quadratically with the population size: this means that a swarm with $3 n$ robots would aggregate 9 times faster than a swarm with $n$ robots. On the contrary, the area of the cue $a_{c}$ would have rather limited impact in the cue aggregation speed, because it mainly influences the aggregation speed in the initial phases, where $p_{m} \gg p_{j}$. The model also suggests that increasing the sensor range $r_{s}$ and robot speed $v_{r}$ will both affect (through the $a_{s}$ ) the aggregation speed in a (linearly) proportional way. In large populations, increasing or decreasing the waiting time should affect the steady number of the aggregated robots only marginally since the chance of a robot escaping the aggregate is low.

\section{Implementation of Aggregation}

\subsection{Robot Platform}

We use Colias (Arvin, Murray, Zhang, \& Yue, 2014) as our robotic platform in our experiments. It is specially designed for swarm applications. It is a small yet capable robot with a diameter of $4 \mathrm{~cm}$. Colias is a compact version of AMiR (Autonomous Miniature Robot) (Arvin, Samsudin, \& Ramli, 2009) with several additional functions enabling the implementation of wide range of swarm behaviors. Fig 2 shows a Colias robot and its modules. The robot has two boards - upper and 
lower - which have different functions. The upper board is for high-level tasks such as inter-robot communication and user programmed scenarios, however, the lower board is designed for low-level functions such as power management and motion control. Two micro DC gearhead motors and two wheels with diameter of $22 \mathrm{~mm}$ move Colias with a maximum speed of $35 \mathrm{~cm} / \mathrm{s}$. The rotational speed for each motor is controlled individually using pulse-width modulation (Arvin \& Bekravi, 2013). Each motor is driven separately by a H-bridge DC motor driver, and consumes power between $120 \mathrm{~mW}$ and $550 \mathrm{~mW}$ depending on the load.

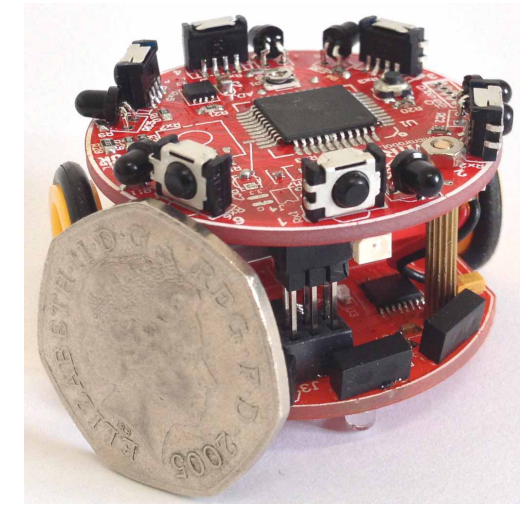

Figure 2: Colias micro mobile robot. The developed platform for swarm robotics research.

Colias uses IR proximity sensors to avoid collisions with obstacles and other robots and a light sensor to detect the intensity of the ambient light. The IR sensing system is composed of two different sub-units: The short-range sensing unit and the long range sensing unit. The short range sensing unit is composed of IR proximity sensors for immediate collision detection in a few centimeters. The long-range sensing unit is composed of six IR proximity sensors (each $60^{\circ}$ on the robot's upper board). It is used for obstacle and robot detection (Arvin, Samsudin, \& Ramli, 2010). It is able to distinguish robots from obstacles within approximately $15 \pm 1 \mathrm{~cm}$. Other than these sensors, Colias has a light (illuminance) sensor at the bottom facing down, which is used to detect illuminance on the ground (this will allow us to use a horizontally placed flat LCD screen as the ground on which robots move, explained in the following section).

In Colias, the lower board is responsible for managing the power consumption as well as recharging process. Power consumption of the robot under normal conditions (in a basic arena with only walls) and short-range communication (low-power IR emitters) is about $2000 \mathrm{~mW}$. However, it can be reduced to approximately $750 \mathrm{~mW}$ when IR emitters are turned on occasionally. A 3.7 V, 


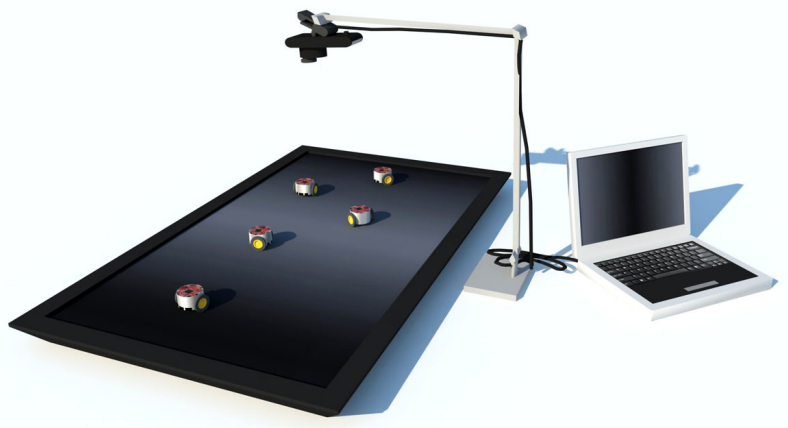

Figure 3: Arena configuration including a 42" LCD screen as the ground and a mounted camera for recording/tracking the experiments.

$600 \mathrm{mAh}$ (extendable up to $1200 \mathrm{mAh}$ ) lithium-polymer battery is used as the main power source, which gives an autonomy of approximately 2 hours for the robot.

\subsection{Arena Setup}

To realize the aggregation experiments, we use rectangular arena with size of $90 \times 57 \mathrm{~cm}^{2}$. We employed a horizontally positioned 42" LCD screen as the ground on which the robots move. Fig. 3 shows the arena setup. In this way, we are able to create complex experiments with different settings with ease. All the aggregation cues, we implemented, are circular light spots with maximum illuminance of 420 lux, which are controlled by a PC.

We use visual localization software developed in (Krajník et al., 2014) to track the robots during experiments using an overhead camera. To reduce the amount of collected data from the localization system, we did not record all experiments in a video. Rather than that, a image of the arena was captured every 20 seconds.

\subsection{Metrics and Statistical Analysis}

We measure the performance of aggregation using the aggregation time, $t_{a}$, and the size of the aggregate, $n_{a}$ metrics. In order to define these two metrics, we need to first define the aggregation zone. The aggregation zone is defined as the area on the cue. A robot waiting on the aggregation zone is regarded an aggregated robot. The aggregation time is defined as the time that the aggregate size reaches at $70 \%$ of the total number of robots. The size of the aggregate is the total number of robots that are in the aggregate at a particular time of the experiment. 
Table 1: Experimental values or range for variables and constants

\begin{tabular}{lll}
\hline Values & Description & Range / Value(s) \\
\hline$n$ & Population size & $\{9,12,15,18\}$ \\
$n_{a}$ & Number of aggregated robots & 0 to 18 robots \\
$n_{f}$ & Number of free robots & 0 to 18 robots \\
$S$ & Sensor reading of illuminance & 0 to 255 \\
$w$ & Waiting time after collision & 0 to $65 \mathrm{sec}$ \\
$v_{r}$ & Robot forward velocity & $7 \mathrm{~cm} / \mathrm{s}$ \\
$r_{s}$ & Radius of robot IR sensory system & $3 \pm 0.3 \mathrm{~cm}$ \\
$r_{r}$ & Radius of robot & $2 \mathrm{~cm}$ \\
$r_{c}$ & Radius of cue & $12 \mathrm{to} 22 \mathrm{~cm}$ \\
$a_{r}$ & Area covered by a robot & $28 \mathrm{~cm}^{2}$ \\
$a_{g}$ & Area covered by swarm & $170 \mathrm{~cm}^{2}$ to $500 \mathrm{~cm}^{2}$ \\
$a_{c}$ & Area of a cue & $300 \mathrm{~cm}^{2}$ to $1500 \mathrm{~cm}^{2}$ \\
$a_{a}$ & Area of the entire arena & $0.51 \mathrm{~m}^{2}$ \\
$v_{c}$ & Motion speed of cue & $\{1,5,10\} \mathrm{mm} / \mathrm{s}$ \\
$t$ & Time & 0 to $800 \mathrm{sec}$ \\
$t_{a}$ & Aggregate time when aggregation is accomplished & 0 to $750 \mathrm{sec}$ \\
$t_{0}$ & Start of an aggregation scenario, $t=0$ & 0 \\
$\beta$ & Ratio of the cue area to the area occupied by swarm & $\{2,2.5,3\}$ \\
\hline
\end{tabular}

All results are statistically analyzed. We used analysis of variance (ANOVA) and the F-test method (Scheaffer, Mulekar, \& McClave, 2010) in the analysis. F-test simply determines the degree of dependency between the selected parameters and results. A high F-value for a parameter means that it has more impact on the result.

The standard values of the constants and variables, which are used in the experiments are listed in Table 1.

\section{Experimental Setup}

\subsection{Static Environment}

In this set of experiments, we study the effects of several parameters; size, texture and number of the cue on aggregation performance in a static manner, i.e., we do not change the settings of an experiment once they are set. Each experiment is repeated with 9, 12, 15, 18 robots.

\subsubsection{Size of Cue}

In this setting, we study the effects of the different cue sizes on the performance of aggregation using a simulated gradient light, i.e. the brightness of the cue gradually decreases from its center. We assume that $a_{r}=\pi r_{s}^{2}$ is the area that a robot has a sensing radius of $r_{s}$ during an instant of 

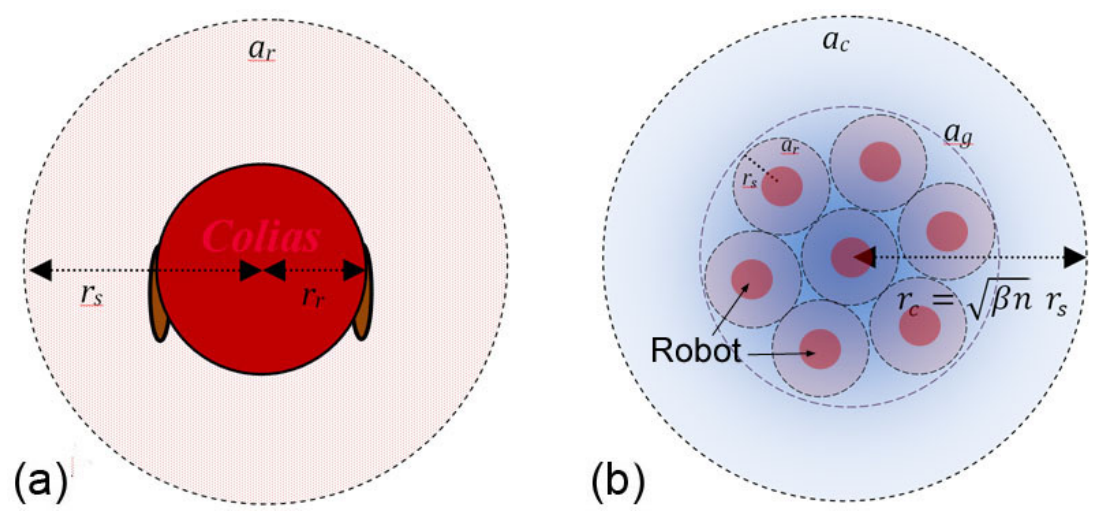

Figure 4: (a) Area, $a_{r}$, which a robot covers using its sensory system with radius of $r_{s}$. (b) A cue implemented with gradient light with an area of $a_{c}$ relative to the number of robots, $n$, and $\beta$.

time (see Fig. 4a). Therefore, the total area which can be covered by radial arrangement of the robots is $a_{g}=n a_{r}$, where $n$ is the number of robots deployed in an experiment.

In these experiments, we use three different sizes of cue for each population size, $a_{c}=\beta n a_{r}, \beta \in$ $\{2,2.5,3\}$ (see Fig. 4b). We increase the size of the cue proportional to the population size. We set the cue sizes from a radius of $12 \mathrm{~cm}$ to $22 \mathrm{~cm}$ based on the population size and $\beta$. In robots, $r_{s}$ is defined to be $3 \pm 0.3 \mathrm{~cm}$ hence $a_{r} \simeq 28 \mathrm{~cm}^{2}$. For example, in case of 9 robots, $a_{g}=250 \mathrm{~cm}^{2}$ so with $\beta=2$ the radius of the cue will be $r_{c}=12 \mathrm{~cm}$, or in case of 18 robots and $\beta=3, a_{g}=540$ $\mathrm{cm}^{2}$ hence the radius of cue will be about $r_{c}=22 \mathrm{~cm}$.

\subsubsection{Texture of Cue}

In these experiments, we study the effect of texture of the cue on aggregation performance. In particular, we formed two types of lighting conditions for the cue. One being the gradient type of lighting and the other being the non-gradient type of lighting. In the gradient cue, the luminance reduces gradually from the center to the edge of the cue, and in the non-gradient cue, the luminance is constant from the center to the perimeter. ${ }^{1}$ For each cue type, we used two different sizes. A small cue with a radius of $r_{c}=16 \mathrm{~cm}$ (1.5 times larger than the area that can accommodate 18 robots) and a large cue with a radius of $r_{c}=20 \mathrm{~cm}(2.5$ times larger than the area that can accommodate 18 robots).

\footnotetext{
${ }^{1}$ Heran et al. showed that honeybee aggregation is not only dependent on temperature itself, but also the temperature gradient around the optimal aggregation zone (Heran, 1952). In order to study this effect in our system, we change the texture, i.e., how light is distributed on the cue.
} 


\subsubsection{Multiple Cues}

In this setting, we study the effect of multiple cues with different sizes on the aggregation performance. In this regard, we used two gradient-type circular cues having different sizes. The main cue (Zone-1 with area of $a_{c_{1}}$ ) has a fixed radius of $r_{c 1}=16 \mathrm{~cm}$ and the size of the second cue (Zone-2 with area of $a_{c_{2}}$ ) is set based on the size of the main cue as: $a_{c_{2}}=k a_{c_{1}}, k \in\left\{\frac{1}{3}, \frac{1}{5}\right\}$. We track the size of the aggregate in both zones. Therefore, an experiment is terminated when the total number of robots (sum of all aggregate sizes) in both zones reaches $70 \%$ of the population size.

\subsection{Dynamic Environment}

In this setting, we change the position of the cue in different ways in order to create a dynamic environment. In particular, we study the adaptability of the swarm to dynamically changing environmental conditions. We created three different sets of experiments in order to test dynamic effects on aggregation performance effectively.

\subsubsection{Switch Cue Location}

In this experiment, a gradient-type cue with the radius of $r_{c}=18 \mathrm{~cm}$ (which is 2 times bigger than the area that can accommodate 18 robots) is used as the aggregation zone. Each run takes 360 sec with three phases, each lasting 120 sec. This value was chosen based on the previous experiments (see Section 7.1) with similar population sizes, where the aggregation time never exceeded $120 \mathrm{~s}$. In the first phase, the cue is placed on the left hand side of the arena. In the second phase of the experiment, the cue is moved instantly to the right hand side of the arena, and in the final phase the cue is moved back to the left hand side instantly. The experiment is performed with two different population sizes of 9 and 18 robots. We record the size of the aggregate during the experiments.

\subsubsection{Delayed Motion}

In this experiment, a single gradient-type circular cue with a radius of $r_{c}=18$ is used. The experiment includes two phases (stationary and moving) each lasting $120 \mathrm{~s}$. In the first phase, the cue is placed on the left hand side of the arena and kept stationary and it starts to move with a 
speed of $v_{c}=3.5 \mathrm{~mm} / \mathrm{s}$ continuously in the second phase. ${ }^{2}$ We repeat the experiment with 12 and 18 robots and we track the size of the aggregate with a period of $20 \mathrm{~s}$.

\subsubsection{Continuous Motion}

In this setting, we use a single gradient-type circular cue with a radius of $r_{c}=18 \mathrm{~cm}$, which moves in a random direction continuously with a speed of $v_{c} \mathrm{~mm} / \mathrm{s}, v_{c} \in\{1,5,10\}$. We repeat the experiment with 9 and 18 robots. During the experiments, we track the number of aggregated robots every $20 \mathrm{~s}$.

\section{Results}

The results of the experiments are presented in this section. The results are depicted as box-plots. In the box-plots, boxes show the range of the first and the third quartiles of the data. Median of the data is shown with a horizontal line inside the boxes. The whiskers show the range between the minimum and maximum values of the data.

A sample video of the swarm behavior and the experimental setup are provided online (Arvin, 2014).

\subsection{Static Environment}

Here, we depict the results of size of cue, texture of cue and multiple cues experiments.

\subsubsection{Size of Cue}

Aggregation time with respect to different cue sizes and number of robots is depicted in Fig. 5 . We can see that for a fixed size cue an increase in the number of robots decreases the aggregation time. This effect is more preeminent when the number of robots is smaller. When we keep the number of robots the same, and change the size of the cue, we observe that larger cue size results in a shorter aggregation time.

These observations are as expected. An increase in the number of robots increases the probability of collisions hence increases the probability to form an aggregate (provided that there is no

\footnotetext{
${ }^{2}$ The reason we chose the speed is that, if a robot encounters another one at a position where the light intensity is high, the robot is going to wait for $55 \mathrm{~s}$. With $r_{c}=18 \mathrm{~cm}$, we guarantee that the waiting robot will not be on the cue after the waiting time is over, since the cue has already moved $19 \mathrm{~cm}$ during the waiting time.
} 
overcrowding effect). On the other hand, an increase in the size of the cue increases the probability of successful collisions (meaning that the collision happened on the cue) hence increasing the probability to form an aggregate. Both resulting in a decrease in aggregation time.

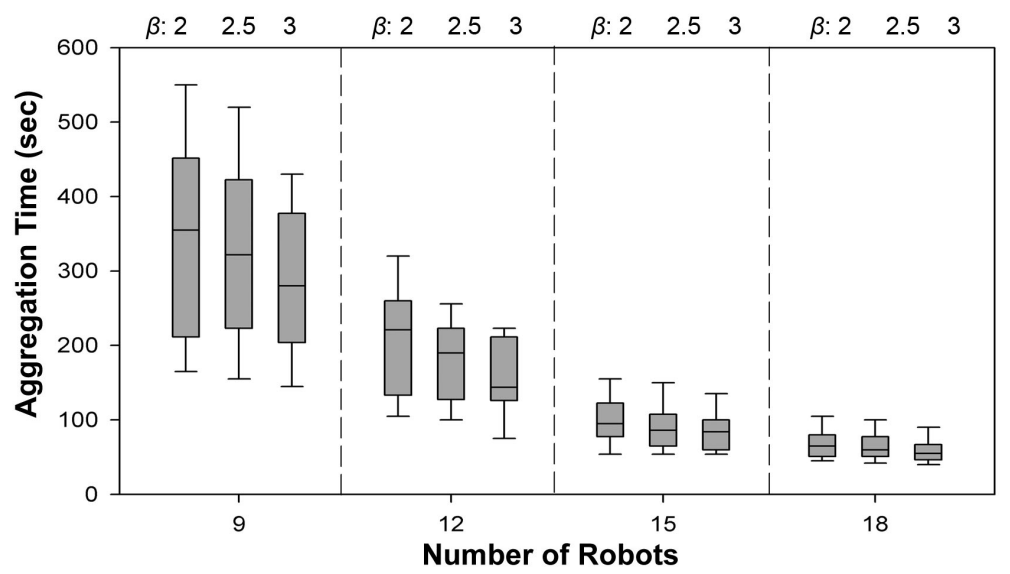

Figure 5: Aggregation time in different population sizes at different cue sizes $\beta \in\{2,2.5,3\}$.

The results of the proposed probabilistic model is depicted (shown in blue continuous line) together with size of cue results (here Fig 5 is redrawn for each $\beta$ ) as in Fig. 6. The model is able to predict aggregation time results both qualitatively and quantitatively.

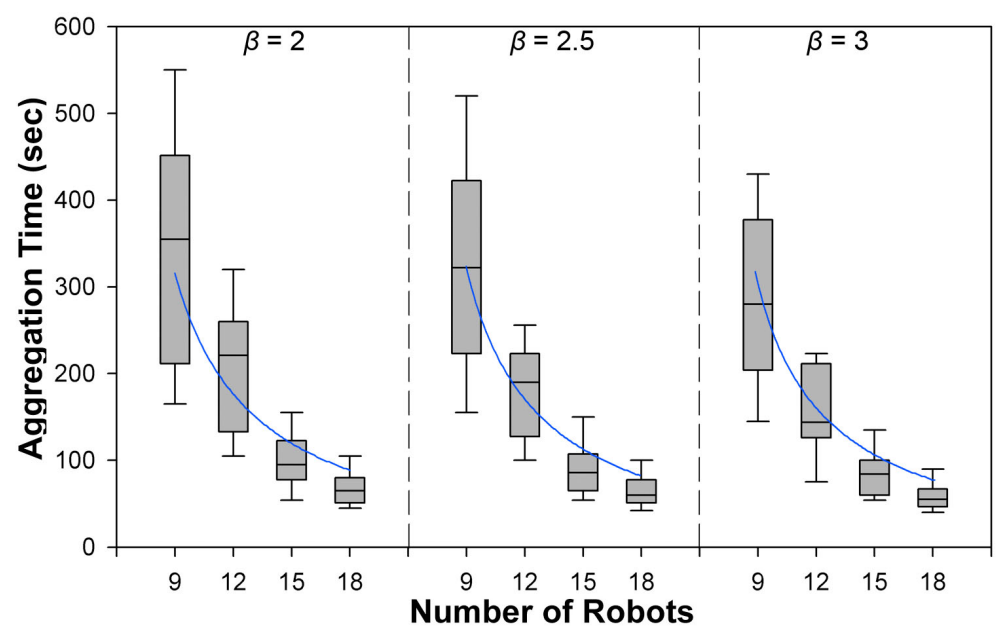

Figure 6: Aggregation time in different $\beta$. Continues lines show the predicted aggregation size by the proposed model.

We analyzed the results statistically. First, we used two-way ANOVA with factors of population and cue sizes to find the most effective factor on the aggregation time. We found that, the population size has more significant influence $(P=0.00, F=81.73)$ than cue size $(P=0.07, F=$ 
2.64) on the aggregation time. We then statistically analyzed the effects of cue size on each population, separately. The results showed that, the changes in cue size affect the aggregation time more in small population than the large population $(F=\{0.85,0.72,0.66,0.51\}$ for $n=$ $\{9,12,15,18\}$, respectively). Therefore, increase in population size compensates the fluctuations in the cue size.

\subsubsection{Texture of Cue}

The results of the experiments with a small cue (top) and a large cue (bottom) depicted in Fig 7 . The predictions of the proposed model is also depicted on the same figure as a continuous blue line. In all the experiments, increase in the number of robots reduces the aggregation time. We can also observe that aggregation times with gradient-type cue is almost the same as the non-gradient-type cue. The only exception is the higher populations (with 15 and 18 robots) with non-gradient cue, which the swarm performance reduced in comparison to the same population size with gradient cue.

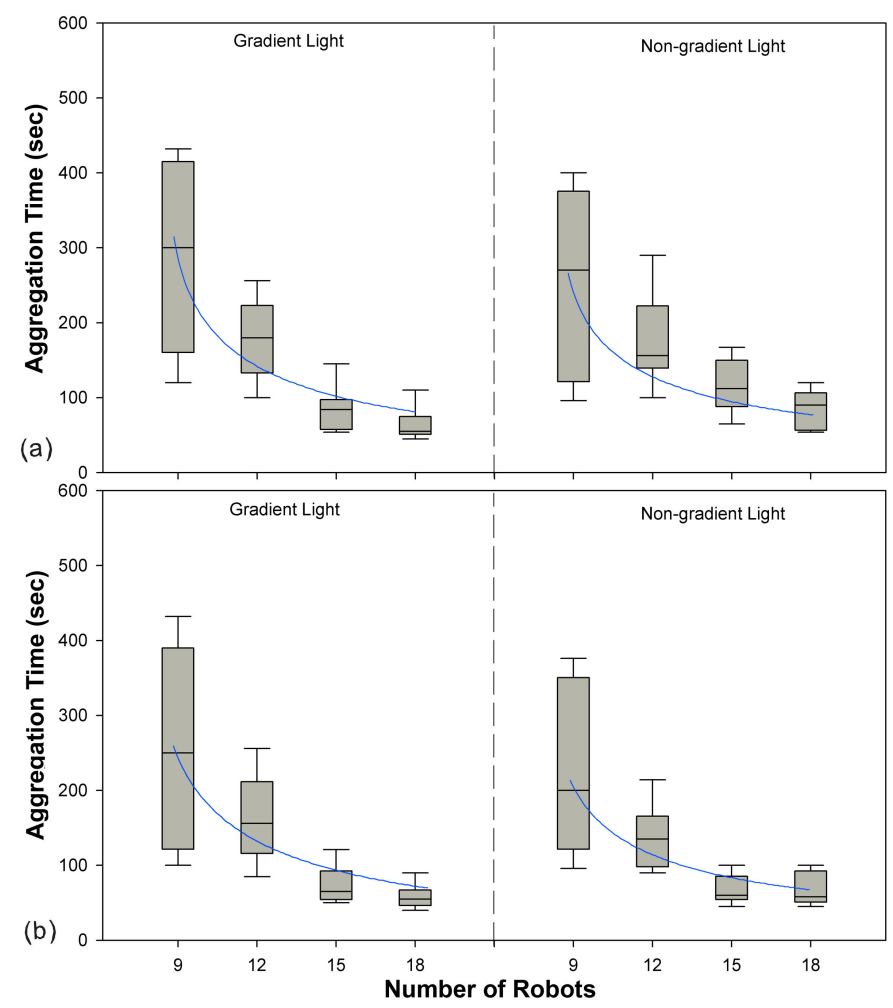

Figure 7: Aggregation time with gradient and non-gradient lights in different population sizes at (a) a small size cue (with radius of $16 \mathrm{~cm}$ ) and (b) a big size cue (with radius of $20 \mathrm{~cm}$ ). Continues lines indicate the predicted values from the probabilistic model. 
Most of the results are in accordance with the expectations. An increase in the number of robots in any setting due to increase in the probability of collisions decreases the aggregation time. Here, we also see this effect. The type of lighting of the cue does not change the performance considerably. This was rather unexpected, but it could be due to geometrical constraints imposed by the size of robots and size of the cue. The proposed model is able to predict the results both qualitatively and quantitatively also in this case.

We also analyzed the results statistically to see how aggregation time is dependent on the different factors. We checked the effects of population and texture of the cue as the factors and the aggregation time as the response (see Table 2). The results of the statistical analysis show that, in both cue sizes, the population size has significant impact on the aggregation time. However, the texture of the cue does not have a significant impact on the performance. We also analyzed the effects of cue size and population as two independent factors. The results of the statistical analysis revealed that, the population size is more effective $(P<0.05, F=77.52)$ than the cue size $(P<0.05, F=9.68)$ on the performance of the swarm.

Table 2: Results of analysis of variance (ANOVA)

\begin{tabular}{llll}
\hline Cue Size & Factor & $P$ value & $F$ value \\
\hline \multirow{2}{*}{ Big cue } & Population & 0.00 & 35.52 \\
& Texture & 0.20 & 1.62 \\
\hline \multirow{2}{*}{ Small cue } & Population & 0.00 & 40.66 \\
& Texture & 0.20 & 1.15 \\
\hline
\end{tabular}

\subsubsection{Multiple Cues}

The results of the experiments with two different cue sizes (second cue is $\frac{1}{3}$ or $\frac{1}{5}$ of the area of the first cue, which has a radius of $16 \mathrm{~cm}$ ) and different population sizes are shown in Fig. 8. We can clearly see that as in all the other experiments, an increase in the population size, decreases the aggregation time. The aggregation time with a large secondary cue (Fig. 8a) is faster than the aggregation time with the smaller secondary cue (Fig. 8b) since we are counting the total number of robots in both zones, bigger second cue means bigger total aggregation zone.

We also investigated the number of aggregated robots at both cues (Zone-1 and Zone-2) separately in varying population sizes as shown in Fig. 9. The results reveal that an increase in the population size increases the size of aggregate at the main cue (Zone-1). However, interestingly the number of the aggregated robots on the small cue (Zone-2) does not show a significant increase. 

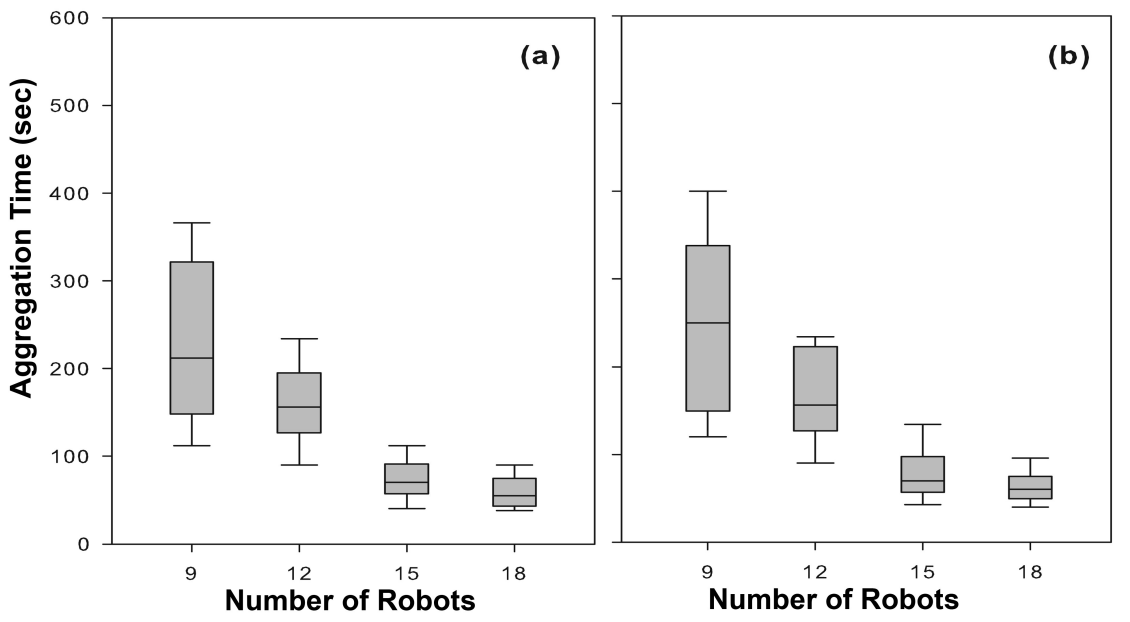

Figure 8: Aggregation time as a function of population size in (a) $a_{c_{2}}=\frac{1}{3} a_{c_{1}}$ and (b) $a_{c_{2}}=\frac{1}{5} a_{c_{1}}$.

In addition, size of the second cue has an impact on the number of aggregated robots on the first cue. The number of aggregated robots increases when the size of the second cue is small.

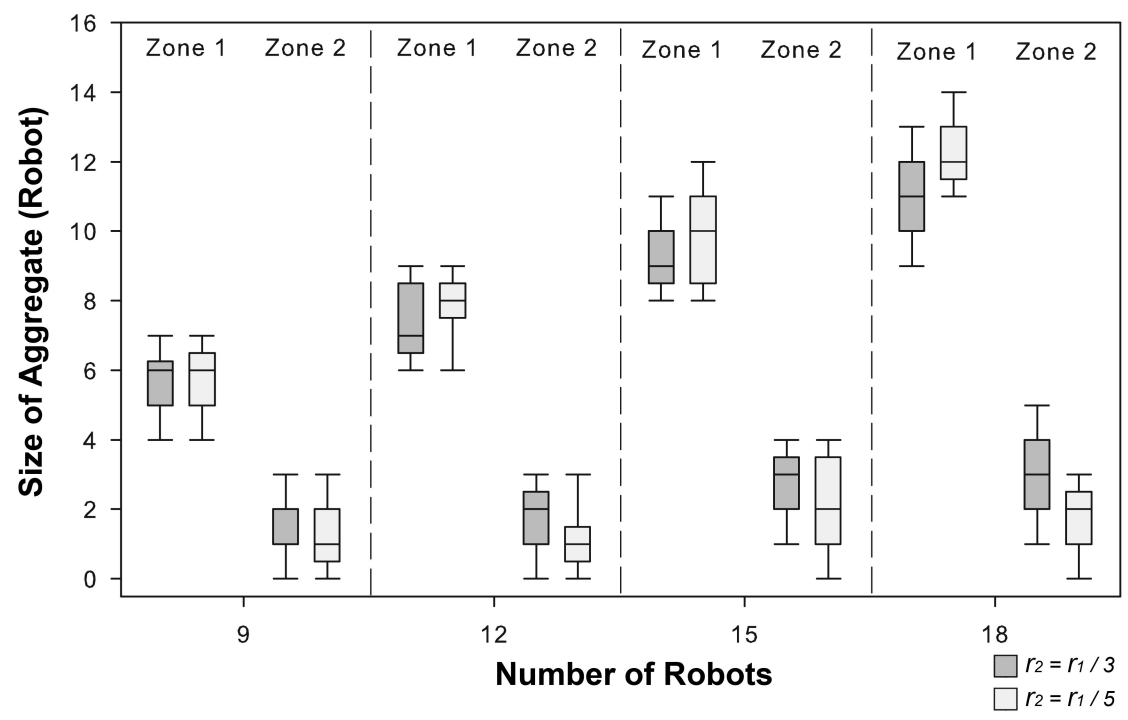

Figure 9: Number of the aggregated robots at Zone-1 (the big size cue with area of $a_{c_{1}}$ ) and Zone-2 (the small size cue with area of $a_{c_{2}}$ ). $a_{c_{2}}=\frac{1}{3} a_{c_{1}}$ (the dark boxes in the diagram) and $a_{c_{2}}=\frac{1}{5} a_{c_{1}}$ (the light boxes in the diagram).

We also statistically analyzed the results (number of robots on Zone-1 and Zone-2 tracked separately) using ANOVA two-way test (see Table 3). The analysis revealed that both population size and the size of the second cue have significant impact $(P<0.05)$ on the number of robots on the first cue. In particular, the population size $(F=214.50)$ influences the size of the aggregate 
on the first cue more than the size of the second cue $(F=42.19)$. We also analyzed the effects of population and cue size as two independent factors. The results of the statistical analysis show that, the population size has more impact $(F=42.81)$ on the aggregation time than the size of the second cue $(F=0.54)$.

Table 3: Results of analysis of variance for size of the aggregate

\begin{tabular}{llll}
\hline Aggregation Zone & Factor & $P$ value & $F$ value \\
\hline \multirow{2}{*}{ Zone-1 } & Population & 0.000 & 214.50 \\
& Ratio of $a_{c_{2}}$ & 0.000 & 42.19 \\
\hline \multirow{2}{*}{ Zone-2 } & Population & 0.005 & 4.06 \\
& Ratio of $a_{c_{2}}$ & 0.001 & 11.31 \\
\hline
\end{tabular}

\subsection{Dynamic Environment}

In this set of experiments, we study the adaptability of the aggregation method to dynamic environments.

\subsubsection{Switch Cue Location}

The time evolution of the size of the aggregate with 12 robots (top) and 18 robots (bottom) are depicted in Fig. 10. During the first $120 \mathrm{~s}$, robots aggregated on the cue, which was on the left hand side of the arena. In the next $120 \mathrm{~s}$, the cue was moved instantly to the right and robots rapidly adapt to the change and start to aggregate on the cue. When the cue was moved back to its original position on the left, the robots again adapted to this change and aggregated on the cue. In general, we can claim that the aggregation method tackled well with dynamically changing cue location.

\subsubsection{Delayed Motion}

The time evolution of the size of the aggregate with two different population sizes (12 robots on the left and 18 robots on the right) is depicted in Fig. 11. During the first $120 \mathrm{~s}$ of the experiment, most of the robots were able to aggregate on the cue. In the second phase, when the cue started to move with a constant speed, we can clearly see that number of aggregated robots decrease slowly and stabilizes around 3 robots for the 12 robots experiment and 7 for the 18 robots experiment around $180 \mathrm{~s}$. We can say that the robots are not able to track a moving cue due to cue's speed $(3.5 \mathrm{~mm} / \mathrm{s})$ 


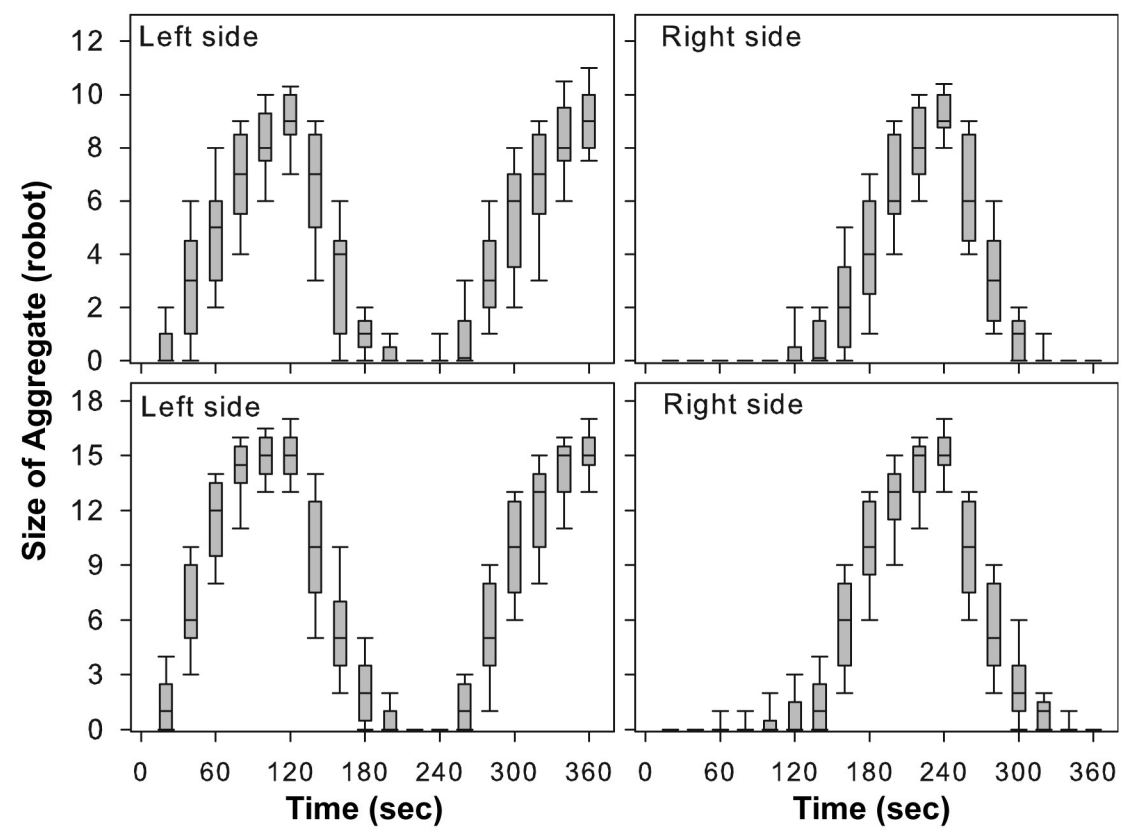

Figure 10: Size of the aggregate during experiments in dynamic environment with different population sizes $(n \in\{12,18\})$.

and high waiting times on the cue. In the next set of experiments, we can clearly see that when cue's speed is low enough $(1 \mathrm{~mm} / \mathrm{s})$, robots are able to track the cue with success.
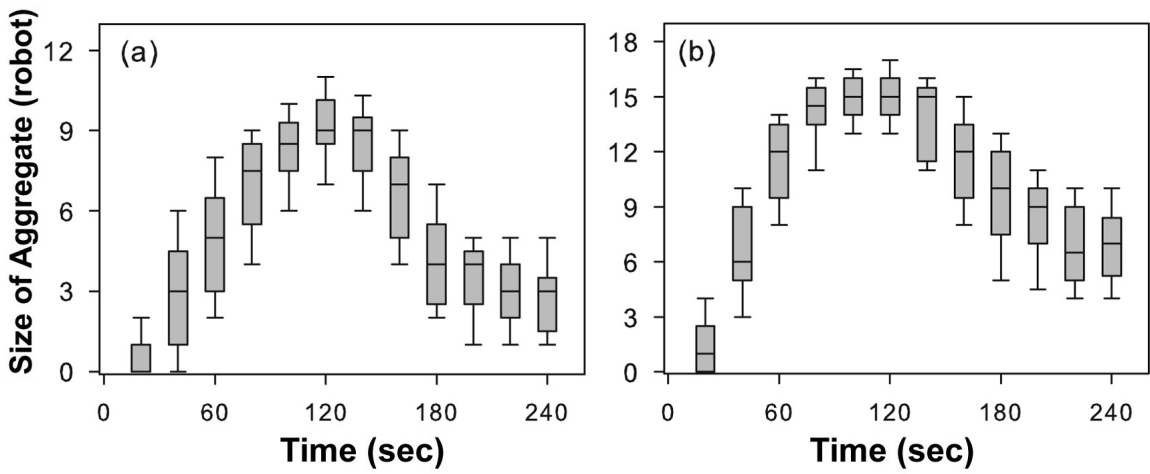

Figure 11: Size of the aggregate during experiments in dynamic environment with different populations $(n \in\{12,18\})$.

\subsubsection{Continuous Motion}

The results of the continuous motion experiment with two different population sizes (9 robots shown with empty boxes, and 18 robots shown with filled boxes) and the prediction of the model (blue continuous line) are depicted in Fig. 12. The results clearly show that the speed of the 
cue, as also discussed in delayed motion experiments, affects the aggregation performance. When the speed is $1 \mathrm{~mm} / \mathrm{s}$, the robots are able to track the cue with considerable success for both population sizes. However, when the speed is $5 \mathrm{~mm} / \mathrm{s}$, the performance decreases considerably and for $10 \mathrm{~mm} / \mathrm{s}$ it is even worse. These results are as expected due to cue speed and waiting time relation. With high waiting times, the robots are not able to cope with high cue speeds, so the aggregation performance is adversely affected. The model is able to predict the size of the aggregate both qualitatively and quantitatively for the two population sizes and the three speeds tested.

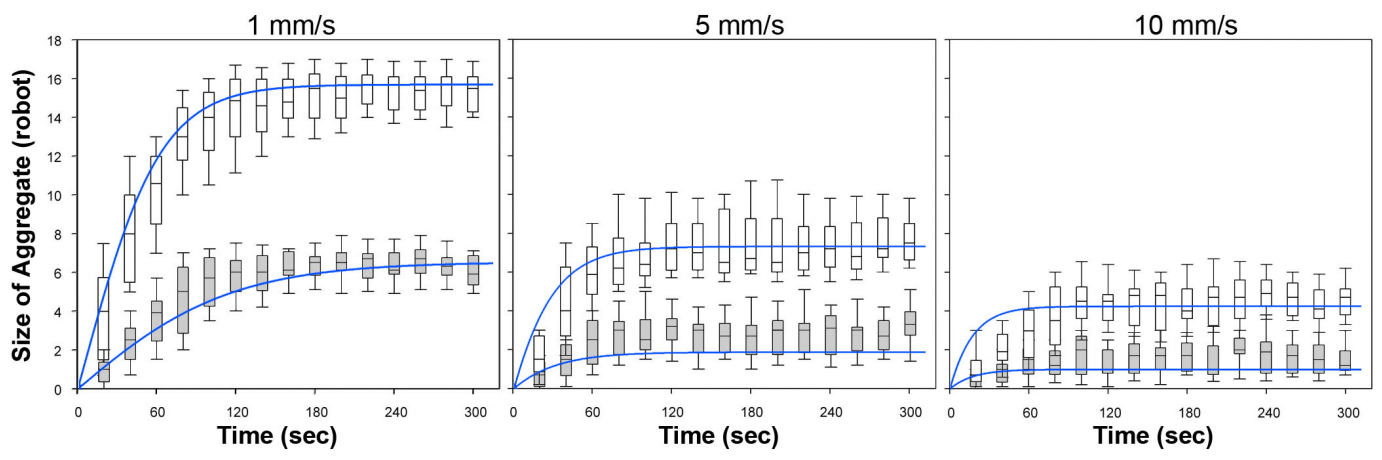

Figure 12: Size of the aggregate during experiments in dynamic environment with different cue speeds $\left(v_{c} \in\{1,5,10\} \mathrm{mm} / \mathrm{s}\right)$. Filled box indicates the results with 9 robots and empty box indicates the results with 18 robots. The continuous lines show the output of model.

\section{Discussion}

The results indicated that environmental changes play a very important role in cue-based aggregation. Any change on the experimental setup changed the aggregation performance. Here, we discuss these effects in the static and dynamic configurations in detail.

\subsection{Static Arena}

Similar to the other works in aggregation (Campo et al., 2011; Arvin et al., 2011; Arvin, Turgut, Bazyari, et al., 2014), an increase in population size increases the performance of the system in the static configuration (Section 7.1) provided that the increase does not cause any interference in the system as noted in (Hamann, 2013). We prevent interference by keeping the number of robots, hence the density of robots below a certain value. Since the BEECLUST method is a collision-based algorithm, any interaction between the robots start with a collision. Increasing 
the number of robots, increases the number of collisions resulting in an increase in aggregation performance. This results is in-line with the fact that increase in the number of agents in a swarm system increases the opportunities of cooperation as discussed in detail in (Hamann, 2013). The following observations are made about the static experiments:

- In the first experiment, we investigated the effect of the cue size on the aggregation performance (Section 7.1.1). The statistical analysis revealed that the population size has a significant impact on the performance. Other than the population size, the size of the cue is also another factor that affects the aggregation performance, which shows itself more in small populations. An increase in the size of the cue increases the probability of successful collisions (collisions on the cue) eventually increasing the performance in low population sizes. The adaptability of a swarm system to the environmental changes was also reported in (Liu, Winfield, Sa, Chen, \& Dou, 2007), which is in accordance with our findings.

- In the second experiment (Section 7.1.2), we tested the effect of the texture of the cue. The aggregation times with the non-gradient and gradient cues are almost the same. Similar to the previously results, increasing the number of robots increases the performance of aggregation. However, the increase in the performance does not remain the same when the population size increases to 15 robots and more due to barrier, which is formed around the cue. Since entire cue has same luminance, in high populations the aggregate is formed nearby the edges hence the way to reach the center of the cue by the other robots is blocked. Statistical analysis of the results showed that the texture and size of the cue have less impact on the the aggregation performance than the population size.

- In the third experiment (Section 7.1.3), we investigated the effect of multiple cues on the aggregation performance. Similar to the other experiments, we first observed that an increase in population size increases the aggregation performance (Fig. 8). We also observed that a large second cue results in a higher performance increase than a smaller second cue, since a large second cue increases the aggregation area more than a smaller cue (in that setting we are counting the total number of robots on the first and second cue). Another observation is that number of robots aggregated on the first cue is more than the second cue, and this difference gets more when the population size increases (Fig. 9). This is a similar phenomenon observed in honeybee aggregation (Szopek et al., 2013) where it is observed that larger groups decide 
faster on optimal temperature zones than smaller groups.

\subsection{Dynamic Arena}

We investigated the adaptation characteristics of the aggregation method by changing the environment conditions dynamically in three different experiments.

- In the first experiment (Section 7.2.1, the location of the cue moves instantly from the leftmost side of the arena to the rightmost side and then moves back to its original position. We observed that similar to the static arena experiments that larger population has higher aggregation performance, which is especially observed in the first phase of the experiments. However, at the start of the second and third phases, robots start to leave the aggregate (the cue has already moved to its next position) when the waiting time is over. Since the waiting time is only a function of light (see Eq. 1), it is not affected by the population size as much as the first phase. A similar behavior in a dynamic environment was also reported in (Liu et al., 2007).

- In the second experiment (Section 7.2.2, we evaluated the adaptability of the aggregation method using a moving cue with a constant speed. The results revealed that the aggregated robots can track the moving cue, but the aggregation performance is not as high as expected. This could be due the speed of the cue, speed of the robots and the duration of the waiting time. Adaptability of a swarm system under various environmental changes has also been studied in (Stewart \& Russell, 2006).

- In the third experiment (Section 7.2.3), the cue moves to a random direction continuously with different speeds. The results showed that when the cue moves with a relatively low speed $(1 \mathrm{~mm} / \mathrm{s})$, the robots can easily track the cue and aggregation performance is high. However, when the speed of the cue increases the robots start to lose the cue as discussed in the second configuration above.

\subsection{Modeling}

The probabilistic model introduced in Section 4 could predict the overall aggregation behavior both qualitatively and quantitatively to an acceptable accuracy, but still it needs to be improved. Some observations are: 
- In the model it is assumed that the robots are uniformly distributed on the cue. However, especially the gradient-type cue causes the aggregated robots to concentrate in the cue center while the non-gradient type cue has most of the aggregated robots around the edge. The distribution of the robots on the cue affects both their waiting times and chances to rejoin the aggregate after their waiting time elapses. Thus, omitting this effect affects the models prediction of the probability that a robot leaves the aggregate.

- The waiting time of the robots is modeled as a probability that a robot leaves at a given time, while the robots wait for a fixed time period. Again, this impacts the model's ability to predict the behavior of the swarm during the initial states of the aggregation.

- The model is inspired by collision modeling of gas molecules, which assumes a specific range of body-per-volume density and molecule speed. We have observed that for low population swarms (6 and below), the model predicted unrealistically long aggregations times.

- The model assumes constant robot speed, but the robots speed vary, e.g. when avoiding the arena walls. This required that the speed of the robots in the model was reduced.

- The model does not represent the effects of sensor noise: Sometimes the robots miss each other even when passing within the sensory range. To represent this effect in the model, we decreased the IR range radius $r_{s}$.

- Some of the environment effects such as the gradient light and sensor noise are difficult to represent rigorously. Thus, we have substituted their effects by parameters that had to be hand-tuned.

Despite of the aforementioned imperfections, the model is able to predict the aggregation times in environments with approximately $10 \%$ error.

\section{Conclusion}

In this paper, we investigated the performance of the state-of-the-art BEECLUST aggregation method in different environmental conditions. We observed that environment plays a very important role in aggregation performance as also observed in social animals such as ants and termites (Depickere, Fresneau, \& Deneubourg, 2008). In particular, we focused on a cue-based 
aggregation scenario and observed some important facts: (1) Despite all the other environmental effects, population size plays the most important role in aggregation performance. Increase in the population size increases the probability of collisions between the robots, hence increases the probability to form an aggregate on the cue. This is an expected result (Campo et al., 2011; Arvin et al., 2011; Arvin, Turgut, Bazyari, et al., 2014) provided that the number of robots (or the density) stays below a critical level in which interference (Goldberg \& Mataric, 1997) starts to occur and degrades the performance (Hamann, 2013). (2) BEECLUST, or in general, a collision-based aggregation method, although being very simple, is able to distinguish between two cues (one being large and the other being small) and more robots aggregate on the larger cue than the smaller cue. This effect is observed even more with a larger population as discussed in Section 8.1. To put in another way, BEECLUST is able to discriminate between two cues (or sources) based on their size (or quality) efficiently in a self-organized way with a very simple decision-making mechanism as observed in social animals (Campo et al., 2011). (3) Adaptation ability of BEECLUST method is quite impressive as observed in dynamic environments (Section 8.2). Unlike other methods (designed purposefully to be adaptive), BEECLUST is inherently adaptive to changing environmental conditions.

As a future work we are planning to investigate the effect of density on aggregation performance. We are planning to test the extreme conditions such as very low density and very high density, and study the effect of interference on system performance using computer-based simulations. We will also study the effects of environmental changes using a heterogeneous swarm and we will look for ways to improve adaptability of the aggregation method by modifying the original method. By solving the differential equation that constitutes our model, we will obtain the aggregate size as a function of time, swarm and environment parameters. This will allow us to infer parameters of individual robots from the global swarm behavior by fitting the model to the observed data.

\section{Acknowledgments}

This work was supported by EU FP7-IRSES projects EYE2E (grant number 269118), LIVCODE (grant number 295151), HAZCEPT (grant number 318907). The third author would thank STRANDS project (grant number 600623). 


\section{References}

Amé, J.-M., Halloy, J., Rivault, C., Detrain, C., \& Deneubourg, J. L. (2006). Collegial decision making based on social amplification leads to optimal group formation. Proceedings of the National Academy of Sciences, 103(15), 5835-5840.

Arvin, F. (2014). BEECLUST with Colias. https://www . youtube. com/watch?v=BurlFPGJIIE.

Arvin, F., Attar, A., Turgut, A., \& Yue, S. (2015). Power-law distribution of long-term experimental data in swarm robotics. In Y. Tan, Y. Shi, F. Buarque, A. Gelbukh, S. Das, \& A. Engelbrecht (Eds.), Advances in Swarm and Computational Intelligence (Vol. 9140, p. 551-559).

Arvin, F., \& Bekravi, M. (2013). Encoderless Position Estimation and Error Correction Techniques for Miniature Mobile Robots. Turkish Journal of Electrical Engineering $\&$ Computer Sciences, 21, 1631-1645.

Arvin, F., Murray, J., Zhang, C., \& Yue, S. (2014). Colias: An Autonomous Micro Robot for Swarm Robotic Applications. International Journal of Advanced Robotic Systems, 11(113), $1-10$.

Arvin, F., Samsudin, K., \& Ramli, A. R. (2009). Development of a Miniature Robot for Swarm Robotic Application. International Journal of Computer and Electrical Engineering, 1(4), 436-442.

Arvin, F., Samsudin, K., \& Ramli, A. R. (2010). Development of IR-Based Short-Range Communication Techniques for Swarm Robot Applications. Advances in Electrical and Computer Engineering, 10(4), 61-68.

Arvin, F., Samsudin, K., Ramli, A. R., \& Bekravi, M. (2011). Imitation of Honeybee Aggregation with Collective Behavior of Swarm Robots. International Journal of Computational Intelligence Systems, 4(4), 739-748.

Arvin, F., Turgut, A. E., Bazyari, F., Arikan, K. B., Bellotto, N., \& Yue, S. (2014). Cue-based aggregation with a mobile robot swarm: a novel fuzzy-based method. Adaptive Behavior, 22, 189-206.

Arvin, F., Turgut, A. E., Bellotto, N., \& Yue, S. (2014). Comparison of different cue-based swarm aggregation strategies. In Advances in Swarm Intelligence (Vol. 8794, pp. 1-8).

Arvin, F., Turgut, A. E., \& Yue, S. (2012). Fuzzy-Based Aggregation with a Mobile Robot Swarm. In Swarm Intelligence (Vol. 7461, p. 346-347). 
Bayındır, L. (2016). A review of swarm robotics tasks. Neurocomputing, 172, 292-321.

Bayindir, L., \& Şahin, E. (2009). Modeling self-organized aggregation in swarm robotic systems. In Swarm Intelligence Symposium (pp. 88-95).

Bonner, J. T. (1944). A Descriptive Study of the Development of the Slime Mold Dictyostelium Discoideum. American Journal of Botany, 31(3), 175-182.

Brambilla, M., Ferrante, E., Birattari, M., \& Dorigo, M. (2013). Swarm robotics: a review from the swarm engineering perspective. Swarm Intelligence, 7(1), 1-41.

Broly, P., Devigne, L., Deneubourg, J.-L., \& Devigne, C. (2014). Effects of group size on aggregation against desiccation in woodlice (isopoda: Oniscidea). Physiological Entomology, 39(2), $165-171$.

Camazine, S., Franks, N., Sneyd, J., Bonabeau, E., Deneubourg, J.-L., \& Theraulaz, G. (2001). Self-organization in Biological Systems. Princeton University Press.

Campo, A., Garnier, S., Dédriche, O., Zekkri, M., \& Dorigo, M. (2011). Self-organized discrimination of resources. PloS ONE, 6(5), e19888.

Correll, N., \& Martinoli, A. (2007). Modeling self-organized aggregation in a swarm of miniature robots. In IEEE International Conference on Robotics and Automation, Workshop on Collective Behaviors Inspired by Biological and Biochemical Systems.

Depickere, S., Fresneau, D., \& Deneubourg, J.-L. (2008). Effect of social and environmental factors on ant aggregation: A general response? Journal of insect physiology, 54 (9), 1349-1355.

Francesca, G., Brambilla, M., Trianni, V., Dorigo, M., \& Birattari, M. (2012). Analysing an evolved robotic behaviour using a biological model of collegial decision making. In International Conference on Simulation of Adaptive Behavior (Vol. 7426, p. 381-390).

Frank, D. D., Jouandet, G. C., Kearney, P. J., Macpherson, L. J., \& Gallio, M. (2015). Temperature representation in the drosophila brain. Nature, 519 (7543), 358-361.

Garnier, S., Gautrais, J., Asadpour, M., Jost, C., \& Theraulaz, G. (2009). Self-Organized Aggregation Triggers Collective Decision Making in a Group of Cockroach-Like Robots. Adaptive Behavior, 17(2), 109-133.

Garnier, S., Jost, C., Gautrais, J., Asadpour, M., Caprari, G., Jeanson, R., ... Theraulaz, G. (2008). The Embodiment of Cockroach Aggregation Behavior in a Group of Micro-robots. Artificial Life, $14(4), 387-408$.

Gauci, M., Chen, J., Li, W., Dodd, T. J., \& Groß, R. (2014). Self-organized aggregation without 
computation. The International Journal of Robotics Research, 33(8), 1145-1161.

Goldberg, D., \& Mataric, M. J. (1997). Interference as a tool for designing and evaluating multirobot controllers. In Aaai/iaai (pp. 637-642).

Grünbaum, D., \& Okubo, A. (1994). Modelling Social Animal Aggregations. In S. Levin (Ed.), Frontiers in Mathematical Biology (Vol. 100, p. 296-325).

Halloy, J., Sempo, G., Caprari, G., Rivault, C., Asadpour, M., Tache, F., .. others (2007). Social Integration of Robots into Groups of Cockroaches to Control Self-organized Choices. Science, 318(5853), 1155-1158.

Hamann, H. (2008). Space-time continuous models of swarm robotics systems: Supporting globalto-local programming (Unpublished doctoral dissertation). Department of Computer Science, University of Karlsruhe.

Hamann, H. (2013). Towards swarm calculus: Urn models of collective decisions and universal properties of swarm performance. Swarm Intelligence, 7(2-3), 145-172.

Heran, H. (1952). Untersuchungen uber den temperatursinn der honigbiene Apis mellifica unter besonderer berucksichtigung der wahrnehmung strahlender warme. Journal of Comparative Physiology A: Neuroethology, Sensory, Neural, and Behavioral Physiology, 34(2), 179-206.

Holland, O., \& Melhuish, C. (1997). An Interactive Method for Controlling Group Size in Multiple Mobile Robot Systems. In 8th International Conference on Advanced Robotics (pp. 201-206).

Jeanson, R., Rivault, C., Deneubourg, J.-L., Blanco, S., Fournier, R., Jost, C., \& Theraulaz, G. (2005). Self-organized Aggregation in Cockroaches. Animal Behaviour, 69(1), 169 - 180.

Johannesen, A., Dunn, A. M., \& Morrell, L. J. (2014). Prey aggregation is an effective olfactory predator avoidance strategy. PeerJ, 2, e408.

Kengyel, D., Hamann, H., Zahadat, P., Radspieler, G., Wotawa, F., \& Schmickl, T. (2015). Potential of heterogeneity in collective behaviors: A case study on heterogeneous swarms. In PRIMA 2015: Principles and Practice of Multi-Agent Systems (Vol. 9387, p. 201-217). Springer International Publishing.

Kernbach, S., Thenius, R., Kernbach, O., \& Schmickl, T. (2009). Re-embodiment of Honeybee Aggregation Behavior in an Artificial Micro-Robotic System. Adaptive Behavior, 17(3), $237-259$.

Krajník, T., Nitsche, M., Faigl, J., Vaněk, P., Saska, M., Přeučil, L., .. Mejail, M. (2014). A Practical Multirobot Localization System. Journal of Intelligent $E$ Robotic Systems, 76(3-4), 
539-562.

Kube, C., \& Zhang, H. (1993). Collective Robotics: From Social Insects to Robots. Adaptive Behavior, 2(2), 189-219.

Lerman, K., Galstyan, A., Martinoli, A., \& Ijspeert, A. (2001). A macroscopic analytical model of collaboration in distributed robotic systems. Artificial Life, 7(4), 375-393.

Liu, W., Winfield, A. F., Sa, J., Chen, J., \& Dou, L. (2007). Towards energy optimization: Emergent task allocation in a swarm of foraging robots. Adaptive Behavior, 15 (3), 289-305.

Liwanag, H. E. M., Oraze1, J., Costa1, D. P., \& Williams, T. M. (2014). Thermal benefits of aggregation in a large marine endotherm: huddling in California sea lions. Journal of Zoology.

Martinoli, A., Ijspeert, A., \& Mondada, F. (1999). Understanding collective aggregation mechanisms: From probabilistic modelling to experiments with real robots. Robotics and Autonomous Systems, 29(1), 51-63.

Mermoud, G., Matthey, L., Evans, W., \& Martinoli, A. (2010). Aggregation-mediated Collective Perception and Action in a Group of Miniature Robots. In International Conference on Autonomous Agents and Multiagent Systems (pp. 599-606).

Morrell, L., \& James, R. (2008). Mechanisms for Aggregation in Animals: Rule Success Depends on Ecological Variables. Behavioral Ecology, 19(1), 193-201.

Parrish, J., \& Edelstein-Keshet, L. (1999). Complexity, Pattern, and Evolutionary Trade-offs in Animal Aggregation. Science, 284(5411), 99-101.

Rappel, W.-J., Nicol, A., Sarkissian, A., Levine, H., \& Loomis, W. F. (1999). Self-organized Vortex State in Two-Dimensional Dictyostelium Dynamics. Physical Review Letters, 83, 1247-1250.

Raveh, S., Vogt, D., Montavon, C., \& Kölliker, M. (2014). Sibling aggregation preference depends on activity phase in the european earwig (forficula auricularia). Ethology.

Şahin, E., Girgin, S., Bayındır, L., \& Turgut, A. E. (2008). Swarm Robotics. In C. Blum \& D. Merkle (Eds.), Swarm Intelligence (Vol. 1, pp. 87-100).

Scheaffer, R. L., Mulekar, M. S., \& McClave, J. T. (2010). Probability and Statistics for Engineers. Cengage Learning.

Schmickl, T., \& Crailsheim, K. (2004). Costs of environmental fluctuations and benefits of dynamic decentralized foraging decisions in honey bees. Adaptive Behavior, 12(3-4), 263-277. 
Schmickl, T., \& Hamann, H. (2011). BEECLUST: A Swarm Algorithm Derived from Honeybees (Y. Xiao \& F. Hu, Eds.). CRC Press.

Schmickl, T., Hamann, H., Worn, H., \& Crailsheim, K. (2009). Two different approaches to a macroscopic model of a bio-inspired robotic swarm. Robotics and Autonomous Systems, 57(9), 913-921.

Schmickl, T., Thenius, R., Moeslinger, C., Radspieler, G., Kernbach, S., Szymanski, M., \& Crailsheim, K. (2009). Get in touch: cooperative decision making based on robot-to-robot collisions. Autonomous Agents and Multi-Agent Systems, 18(1), 133-155.

Soysal, O., Bahçeci, E., \& Şahin, E. (2007). Aggregation in swarm robotic systems: Evolution and probabilistic control. Turkish Journal of Electrical Engineering 83 Computer Sciences, $15(2), 199-225$.

Soysal, O., \& Şahin, E. (2007). A macroscopic model for self-organized aggregation in swarm robotic systems. In E. Şahin, W. Spears, \& A. Winfield (Eds.), Swarm Robotics (Vol. 4433, p. 27-42).

Soysal, O., \& Şahin, E. (2005). Probabilistic aggregation strategies in swarm robotic systems. In Swarm Intelligence Symposium (pp. 325-332).

Stewart, R. L., \& Russell, R. A. (2006). A distributed feedback mechanism to regulate wall construction by a robotic swarm. Adaptive Behavior, 14(1), 21-51.

Szopek, M., Schmickl, T., Thenius, R., Radspieler, G., \& Crailsheim, K. (2013). Dynamics of collective decision making of honeybees in complex temperature fields. PloS ONE, 8(10), e76250

Trianni, V., Groß, R., Labella, T. H., Şahin, E., \& Dorigo, M. (2003). Evolving Aggregation Behaviors in a Swarm of Robots. In Advances in Artificial Life (Vol. 2801, p. 865-874).

\section{Appendix A}

To be able to adjust the parameters of the probabilistic model easily while having a good overview of their influence on the swarm behavior, we have created a SIMULINK model of the Eq. 7. The model breaks down the Eq. 7 to the individual products and sums of the model parameters (robot speed, sensor range, arena dimensions, cue area) and the number of currently aggregated and nonaggregated robots. The results of the calculation, which are the rates at which the robots leave and join the aggregate, are summed and passed to the integrator block, that represents the system 
state - the number of aggregated robots. In other words, the SIMULINK model is equal to the integral form of the differential Eq. 7. Fig. 13 shows the SIMULINK model.

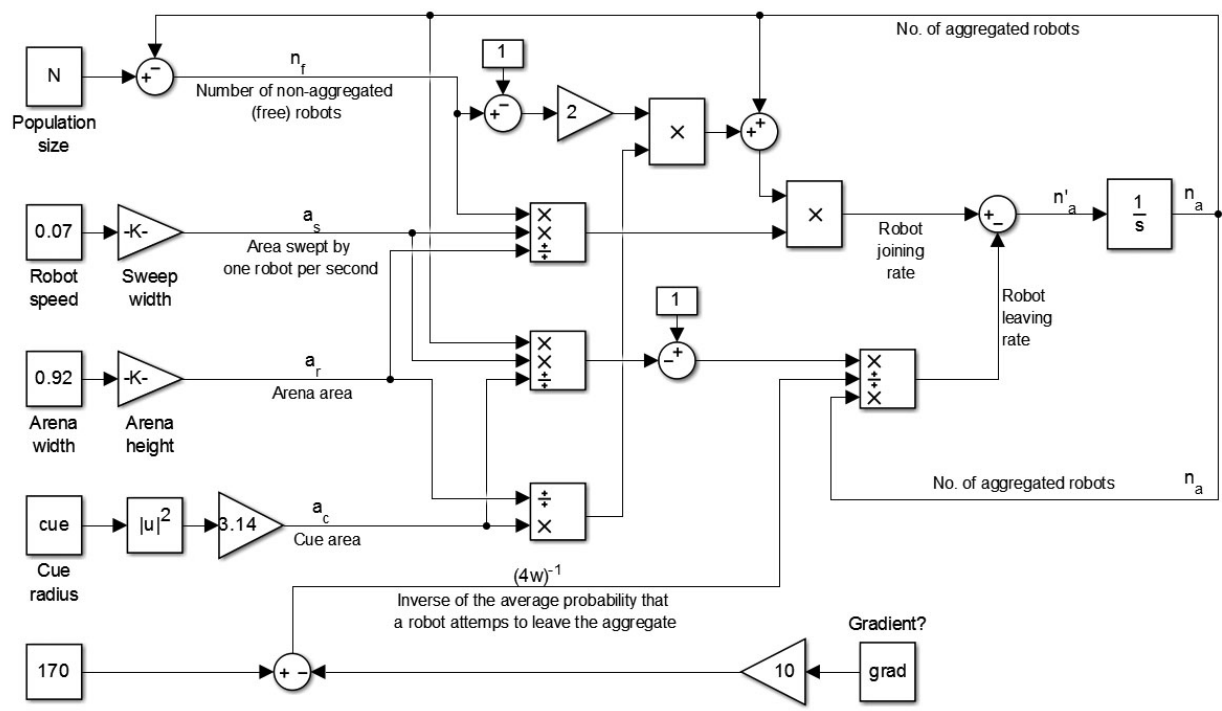

Figure 13: SIMULINK model for the probabilistic modeling of the aggregation.

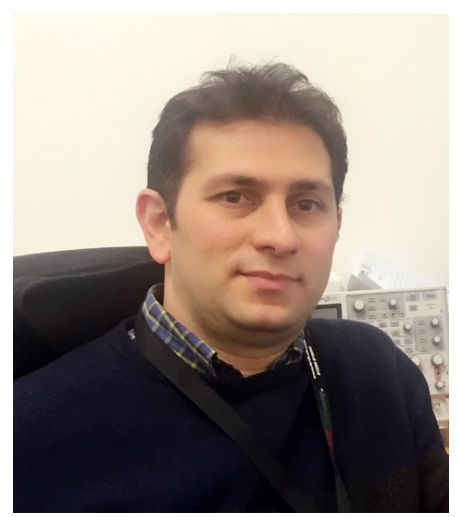

Farshad Arvin is a Research Associate at the School of Electrical and Electronic Engineering at The University of Manchester, UK. He holds a Ph.D. in Computer Science from University of Lincoln, UK. He was research assistant at the Computational Intelligence Laboratory (CIL) at the University of Lincoln under the supervision of Professor Shigang Yue. He received his B.Sc. degree in Computer Engineering and M.Sc. degree in Computer Systems Engineering in 2004 and 2010, respectively. His research interests include autonomous robots, swarm robotics and signal processing. He was awarded a Marie Curie Fellowship to be involved in the FP7- EYE2E and LIVCODE projects during his Ph.D. study. He visited the Institute of Microelectronics at 
Tsinghua University in Beijing, China from September 2012 to August 2013 as a senior scholar under the supervision of Professor Zhihua Wang. He visited the Institute of Rehabilitation and Medical Robotics in Huazhong University of Science and Technology (HUST), Wuhan, China in 2014 under the supervision of Professor Caihua Xiong.

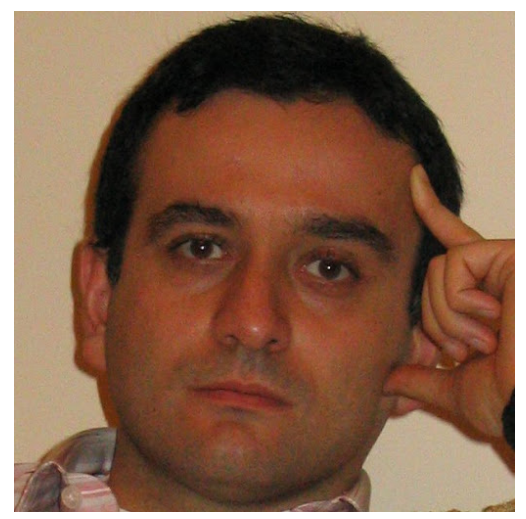

Ali Emre Turgut has received a B.Sc. in mechanical engineering from Middle East Technical University, Turkey, in 1996, a M.Sc. in mechanical engineering from Middle East Technical University, Turkey, in 2000 and Ph.D. in mechanical engineering from Middle East Technical University at Kovan Research Laboratory, Turkey, in 2008. He worked as a post-doctoral researcher at Universite Libre de Bruxelles, IRIDIA, Belgium and as a research associate at the department of biology at KU Leuven, Belgium during 2008-2012. In 2013, he worked as an assistant professor in the Department of Mechatronics Engineering in University of Aeronautical Association of Turkey. He is currently working as a research associate in Laboratory of Socioecology and Social Evolution, KU Leuven. He started working in Mechanical Engineering Department at METU as an assistant professor in 2015 .

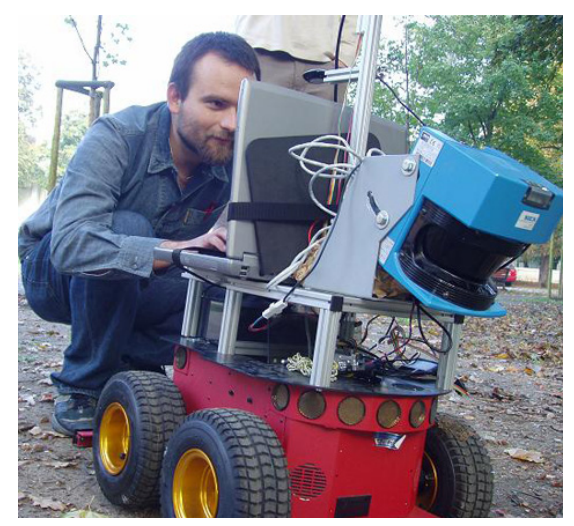

Tomáš Krajník is a research fellow at the Lincoln Center of Autonomous Systems, UK. He has 
received the Ph.D. degree in Artificial Intelligence and Biocybernetics from the Czech Technical University, Prague, Czech Republic, in 2012. His research interests include life-long autonomous navigation, spatio-temporal mapping, and aerial robots.

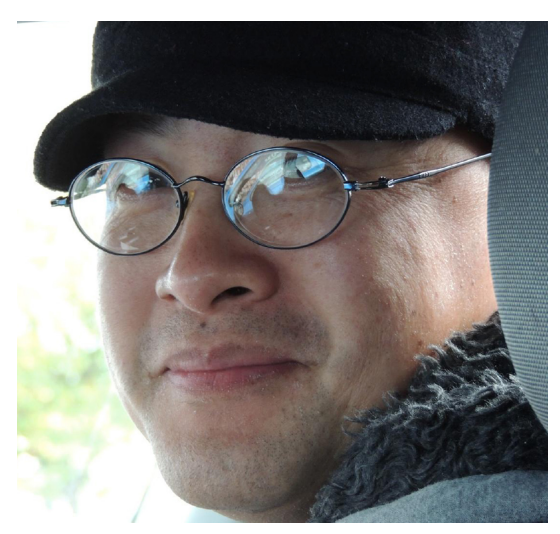

Shigang Yue is a Professor in the School of Computer Science, University of Lincoln, United Kingdom. He received PhD and MSc degrees from Beijing University of Technology (BJUT) in 1996 and 1993, and BEng degree from Qingdao Technological University (1988). He worked in BJUT as a Lecturer (1996-1998) and an Associate Professor (1998-1999). He was an Alexander von Humboldt Research Fellow (2000, 2001) at the University of Kaiserslautern, Germany. Before joining the University of Lincoln as a Senior Lecturer (2007) and promoted to Reader (2010) and Professor (2012), he held research positions in the University of Cambridge, Newcastle University and the University College London (UCL) respectively. His research interests are mainly within the field of artificial intelligence, computer vision, robotics, brains and neuroscience. He is particularly interested in biological visual neural systems, evolution and coordination of neuronal subsystems, ecosystems and their applications e.g., in collision detection for vehicles, interactive systems and robotics. He is the founding director of Computational Intelligence Laboratory (CIL) in Lincoln. He is the coordinator for several EU FP7 projects. He is a member of IEEE, INNS, ISAL and ISBE. 